\title{
THERMODYNAMICAL DESCRIPTION OF HEAVY ION COLLISIONS
}

\author{
T. GAITANOS, H. H. WOLTER \\ Sektion Physik, Universität München, Am Coulombwall 1 \\ D-85748 Garching, Germany \\ E-mail:Theo.Gaitanos@Physik.uni-muenchen.de \\ C. FUCHS \\ Institut für Theoretische Physik, Universität Tübingen, Auf der Morgenstelle 14 \\ D-72076 Tübingen, Germany
}

\begin{abstract}
We analyze the thermodynamical state of nuclear matter in transport descriptions of heavy ion reactions. We determine thermodynamical variables from an analysis of local momentum space distributions and compare to blast model parameters from an analysis of fragment energy spectra. These descriptions are applied to spectator and fireball matter in semi-central and central $\mathrm{Au}+\mathrm{Au}$ collisions at SISenergies, respectively.
\end{abstract}

A topic of great interest in the study of intermediate energy heavy-ion collisions is the investigation of the nuclear equation of state away from saturation at lower densities andat finite temperature in connection to possible

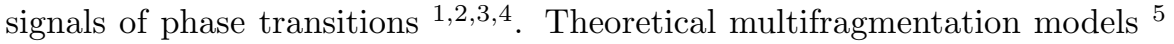
have addressed this question, but their application in the drnamical situation of heavy-ion collisions is difficult due to non-equilibrium 6 and finite size effects 6 . The question is whether the nuclear matter in heavy-ion collisions is both in thermodynamical equilibrium and instable, i.e. whether the fragment emitting source can be characterized by a thermodynamically well defined freeze-out configuration. In this work we investigate this question by studying the results of transport calculations described in detail in Refs. have applied thermodynamical analyses to spectator and participant matter using either the information of the local momentum distribution to define a local thermodynamical temperature $\left(T_{l o c}\right)$ or from a blast model analysis 10 of fragment energy spectra to obtain a slope parameter $\left(T_{\text {slope }}\right)$ of the fragment spectra. We compare these "theoretical data" with experiments.

Fig. 1 on the left shows the temporal evolution of the local temperature $T_{l o c}$ in spectator matter for semicentral $(b=4.5 \mathrm{fm}) \mathrm{Au}+\mathrm{Au}$ reactions at different energies, as obtained from fits to the local momentum space distribution of transport calculations. After the spectators are well defined $T_{l o c}$ approaches rather constant values and remains fairly stable for several $\mathrm{fm} / \mathrm{c}$. The local 

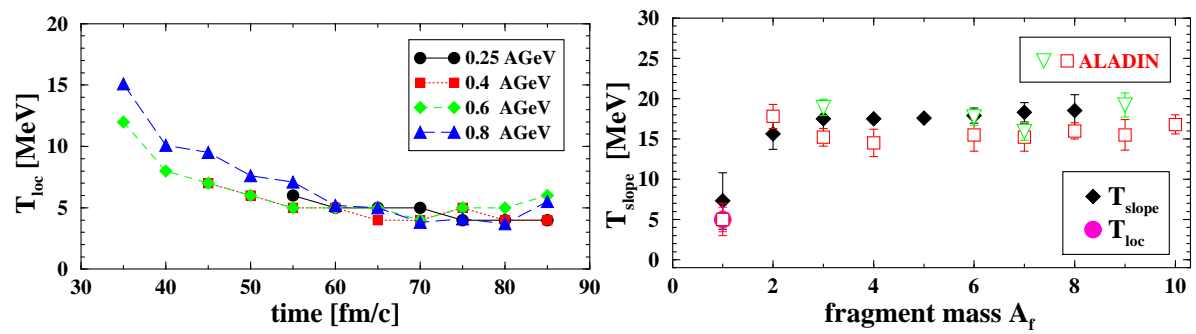

Figure 1. Left: local temperatures $T_{l o c}$ as function of time in spectators for semi-central $\mathrm{Au}+\mathrm{Au}$ reactions. Right: slope temperatures $T_{\text {slope }}$ for spectator fragments as function of $A_{f}$ with comparison to ALADIN data 3. The local temperature of nucleons $T_{l o c}$ is also shown.

temperature is independent on the incident energy indicating the existence of an intermediate equilibrium state. By considering an effective compressibility $K \sim \partial P / \partial \rho$ 目 and instability by $K<0$ we find that after $t \gtrsim 40-45 \mathrm{fm} / \mathrm{c}$ the spectator enterns into an instability region $\mathrm{O}$ and therefore may break up into fragments. The breakup conditions of $T_{l o c} \sim 5-6 \mathrm{MeV}$ and $\rho \sim\left(\frac{1}{3}-\frac{1}{2}\right) \rho_{\text {sat }}$ are consistent with experimental isotope temperatures and densities extracted from 2-particle correlations 3.11 . The generation of fragments by a phase space coalescence in the spectator matter and an analysis of the fragment energy spectra in the blast model 10 leads to the results of Fig. 1 on the right. The independence of $T_{\text {slope }}$ on $A_{f}$ indicates that the fragments are emitted from an equilibrated source. However, the slope temperature of the fragments is higher compared to $T_{\text {slope }}$ of the nucleons, which in turn is equal to $T_{l o c}$. This is understood by the Fermi-motion of the nucleons in the fragmenting source in the spirit of the Goldhaber model, as discussed in Refs. 3.12.13. The slope parameter of the fragments is thus consistent with the nucleon $T_{\text {slope }}$ and also with the local temperature $T_{l o c}$. We thus conclude the existence of a common freeze-out configuration for nucleons and fragments in spectator decay. This interpretation is also supported by the experiments 3 shown in the Fig. 1.

The results of the same analysis for participant matter in central heavyion reactions are summarized in Fig. 2 where a radial flow velocity $\beta_{f}$ appears as an additional parameter in the blast model fits due to the radial expansion of the fireball. We observe that slope temperatures rise and flow velocities fall with increasing fragment mass in contrast to the behavior for spectator fragments in Fig. 1. A similar hehavior has been seen experimentally at $1 \mathrm{AGeV}$ in 14 and theoretically in 15,16 . This behavior cannot be interpreted as fragments originating from a common freeze-out state, i.e. from a unique fragmenting 


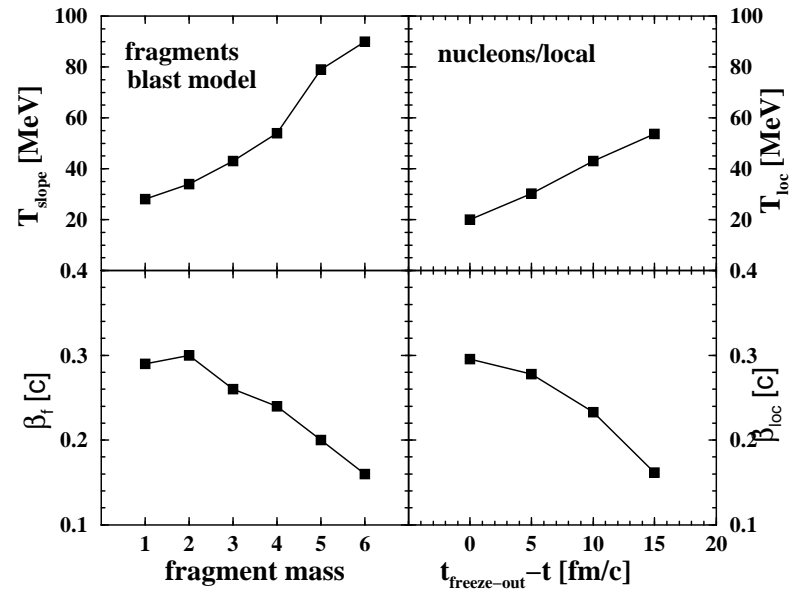

Figure 2. (left) Slope temperatures and radial flow as function of fragment mass. (right) Local values from the momentum distributions at times before the freeze-out for a central $\mathrm{Au}+\mathrm{Au}$ reaction at $0.6 \mathrm{AGeV}$.

source. To arrive at an interpretation we have compared these results with the local temperatures and flow velocities for different times before the nucleon freeze-out, i.e. for $t^{\prime}=t_{\text {freeze-out }}-t$ with $t_{\text {freeze-out }} \sim 35 \mathrm{fm} / \mathrm{c}$. It is seen that for $A_{f}=1$ the values at freeze-out are close to the blast model ones, as required. However, for fragment masses $A_{f}>1$ the slope temperatures and velocities behave qualitatively very similar to the local temperatures and flow velocities at earlier times. This would suggest to interpret the fragment temperatures and velocities as signifying that heavier fragments originate at times earlier than the nucleon freeze-out. This may not be unreasonable since in order to make a heavier fragment one needs higher densities which occur at earlier times and hence higher temperatures. However, this does not neccessarily imply that the fragments are really formed at this time, since fragments could hardly survive such high temperatures, as also discussed in refs. 10.t 0 . But it could mean that these fragments carry information about this stage of the collision. In any case it means that in the participant region fragments are not formed in a common equlibrated freeze-out configuration, and that in such a situation slope temperatures have to be interpreted with great caution.

In summary fragmentation phenomena in heavy ion collisions are studied as a means to explore the phase diagram of hadronic matter. For this it is neccessary to determine the thermodynamical properties of the fragmenting source. One way to do this experimentally is to investigate fragment kinetic 
energy spectra. In theoretical simulations the thermodynamical state can be obtained locally in space and time from the phase space distribution. In this work we have compared this with the information obtained from the generated fragment spectra. We apply this method to the spectator and participant regions of relativistic $\mathrm{Au}+\mathrm{Au}$-collisions. We find that the spectator represents a well developed, equilibrated and instable fragmenting source. In the participant region the local temperature at the nucleon freeze-out and the slope temperatures from fragment spectra behave differently from those of the spectator. The slope temperatures rise with fragment mass which might indicate that the fragments are not formed in a common, equilibrated source.

\section{References}

1. J. Richert, P. Wagner, nucl-th/0009023 (to appear in Phys. Rep.).

2. J. Pochodzalla, Prog. Part. Nucl. Phys. 39, 443 (1997).

3. T. Odeh et al., Phys. Rev. Lett. 84, 4557 (2000).

4. Proc. of the International Workshop XXVII on Gross Properties of Nuclei and Nuclear Excitations, Hirschegg, Austria, 1999.

5. J.P. Bondorf et al., Nucl. Phys. A 443, 321 (1985);

A. S. Botvina, D.H.E. Gross, Nucl. Phys. A 592, 257 (1995) and contribution to this conference.

6. H.H. Wolter, C. Fuchs, T. Gaitanos, Prog. Part. Nucl. Phys. 42, 137 (1999);

T. Gaitanos, C. Fuchs, H.H. Wolter, Nucl. Phys. A 650, 97 (1999).

7. PH. Chomaz, F. Gulminelli, Nucl. Phys. A 647, 153 (1999);

M. D'Agostino et al., Phys. Lett. B 473, 219 (2000) and contribution to this conference.

8. C. Fuchs, P. Essler, T. Gaitanos, H.H. Wolter, Nucl. Phys. A 626, 987 (1997).

9. T. Gaitanos, H.H. Wolter, C. Fuchs Phys. Lett. B 478, 79 (2000).

10. P.J. Siemens, J.O. Rasmussen, Phys. Rev. Lett. 42, 880 (1979);

W. Reisdorf et al., Nucl. Phys. A 612, 493 (1997).

11. S. Fritz et al., Phys. Lett. B 461, 315 (1999).

12. A. S. Goldhaber, Phys. Lett. B 53, 306 (1974); Phys. Rev. C 17, 2243 (1978).

13. W. Bauer, Phys. Rev. C 51, 803 (1995).

14. M. Lisa et al., Phys. Rev. Lett. 75, 2662 (1995).

15. F. Daffin, K. Haglin, W. Bauer, Phys. Rev. C 54, 1375 (1996).

16. A. Hombach et al., Eur. Phys. J. A 5, 157 (1999).

17. W. Neubert, A.S. Botvina, Eur. Phys. J. A 7, 101 (2000).

bologna2: submitted to World Scientific on October 30, 2018 